ORIGINAL ARTICLE

\title{
Urban legend versus rural reality: patients' experience of attendance at accident and emergency departments in west Wales
}

\author{
C D Palmer, K H Jones, P A Jones, S V Polacarz, G W L Evans
}

Emerg Med J 2005;22:165-170. doi: 10.1136/emj.2003.007674

See end of article for authors' affiliations

Correspondence to: Mr G W L Evans, Consultant in A\&E, Pembrokeshire \& Derwen NHS Trust, Withybush General Hospital, Fishguard Road, Haverfordwest SA61 2PZ, UK; gwlevans@rcsed.ac.uk

Accepted

10 November 2003

\begin{abstract}
Objectives: To investigate why and how patients decide to attend accident and emergency (A\&E) departments, and to assess their satisfaction with the experience, in a predominantly rural west Wales population.

Methods: This was a semi-structured follow up telephone interview of patients who walked in to A\&E in one of four general hospitals in west Wales and were triaged as Manchester Triage score 4 or 5. Patients were recruited by nurses during the period July-November 2002. The study sample consisted of 176 male and 145 female patients, mean (SD) age 36.6 (20.0) years. The main outcome measure was a quantitative and qualitative description of the recalled experiences of A\&E attenders, the circumstances of their attendance, and their satisfaction with the experience.

Results: Of the study sample, $78 \%$ attended with injury or illnesses of recent origin, and $50 \%$ with actual or presumed musculoskeletal injury, $73 \%$ of which were sustained within 10 miles of home. Travel to hospital was by private transport for $86 \%$, average distance 7.4 miles. The majority (90\%) were registered with a local GP, but $32 \%$ felt A\&E was the obvious choice, and a further $44 \%$ considered their GP inaccessible to their needs. Patients' reasons for seeking health care at A\&E were similar to those described in an English urban study. Waiting times were rarely excessive; $80 \%$ left within 2 hours, and patient satisfaction was generally high. Among the 87 patients (27\%) who reported a less satisfactory experience, 48 (55\%) of these complained of dismissive attitudes of doctors.

Conclusions: Anecdotal accounts of abuse of A\&E services and unreasonable patient expectations gain the status of "urban legends" within the medical profession. Among the predominantly settled rural population in west Wales, there is little evidence of unreasonable patient expectations, and most patients report high satisfaction levels. Patients' bad experiences most frequently arise from a dismissive attitude on the part of medical staff. These attitudes are often consequent on an A\&E culture that views some patients' attendances as less appropriate than others.
\end{abstract}

t has been a long held view that a substantial proportion of patients presenting at accident and emergency (A\&E) departments is there inappropriately, and that their condition should have been dealt with in primary care, by a visit to their general practitioner (GP). ${ }^{1}$ Patients, however, often choose to go to A\&E. In the wake of the Tomlinson Report on London Health Services, ${ }^{2}$ GP services were introduced on an experimental basis at several urban A\&E departments, following the model already in place at King's College Hospital, London. Research from King's College School of Medicine and Dentistry, London, ${ }^{3-5}$ and from North Thames, ${ }^{6}$ Glasgow, ${ }^{7}$ and Dublin, ${ }^{8}$ has made a strong case for the efficacy of a GP treatment stream within A\&E departments. It has also cast doubt upon the usefulness of the term "inappropriate attender" and upon the prospect of altering patients' use of the services. ${ }^{9}{ }^{10}$ While in urban areas, A\&E attenders more eligible for primary care are often socially deprived, homeless, or destitute, ${ }^{11-13}$ in rural areas the distance from home to hospital, and factors such as time of day, GP surgery hours, and availability of transport are important. ${ }^{14}$ is

At the commencement of this research, A\&E consultants at the four west Wales hospitals involved estimated that the proportion of "inappropriate attenders" comprised 6-30\% of the workload, similar to those reported in recent literature. ${ }^{16}$ In addition, apocryphal stories of patients' unreasonable expectations or inconsiderate behaviour are a part of the culture of accident and emergency. ${ }^{17}$ Such has long been the case in A\&E departments throughout the world; "everyone from clerks to departmental chairmen relate a litany of abuses". ${ }^{18}$ Anecdotally, west Wales "legends" include that of the family who summoned an ambulance for a non-life threatening injury, and then all drove themselves to hospital behind the ambulance to keep the patient company. It is sometimes claimed that holidaymakers save up their nonurgent problems until they arrive in west Wales, as they judge (with reason) that waiting times at those A\&E departments will be much shorter than they would be in their metropolitan hospitals. Casualty departments were renamed as accident and emergency departments in response to the Platt Report of $1962,{ }^{19}$ in the hope of emphasising the immediate nature of the health problems that such departments were expected to treat. Staff may therefore be inclined to impatience with patients who present with a long standing condition that, although it may have recently intensified, is a part of a complaint that may have troubled them for many years.

This study was proposed to gain insight into the behaviour of "inappropriate attenders" in a predominantly rural area and the decision process by which patients came to attend at A\&E departments. However, the design of the project was challenged by the results of a joint nursing audit of patients graded 5 (or Blue) according to the Manchester Triage Score,

Abbreviations: A\&E, Accident and Emergency department; GP, general practioner 
who attended the four A\&E departments. ${ }^{20}$ This audit revealed that, in the opinion of senior nurses, only $1.3 \%$ of patients could genuinely and retrospectively be viewed as inappropriate. Even among these, a closer inspection of the case often revealed good reason, from the patients' perspective, for choosing to attend A\&E. The results of this preliminary study correspond with the findings of Walsh, ${ }^{21}$ that patients "foraging" for health care make logical and efficient choices, and that even if they are viewed by the organisation as a problem, it may be difficult to deter them. It has already been shown by extensive literature review, ${ }^{22}{ }^{23}$ that there is no consistent definition of the inappropriate attender, and that nursing staff differ from other health professionals in considering the majority of patients to be, in fact, attending the appropriate place for the treatment of their condition.

The emphasis of the study thus moved from the study of definably inappropriate attenders (who are few in number) to an investigation into the behaviour and opinions of triage 4 and 5 patients, broadly categorised as the "walking wounded". The study meets the objective set out by the Audit Commission ${ }^{24}$ that hospitals should take proactive steps to ascertain the opinion of patients about their experiences of A\&E, as it is recognised that neither letters of formal complaint nor letters of appreciation represent the spectrum of patient experience.

\section{METHODS}

The project was initially confined to patients categorised as triage 5. However, the recruitment was later extended to include triage 4 because of the small numbers of category 5 individuals. The researcher made contact with patients subsequent to their attendance to conduct a semi-structured telephone interview designed to elicit a full account of the patient's problem, decision making process, means of attending hospital, and experience of and satisfaction with treatment. Some questions were framed for comparability with the research of Walsh. ${ }^{21}$ However, while Walsh interviewed patients who were waiting for treatment, the present study allowed a retrospective judgement on the part of the patient and elicited it in a setting in which the patient should feel less insecure about expressing any dissatisfaction they may feel.

Local research ethics committee and Caldicott guidelines prevented the researcher from inspecting casualty card records or contacting patients, unless their explicit written consent had been obtained. As such, the assembly of the sample depended on the co-operation of triage nurses in asking patients to be a part of the study. Nurses offered a patient information sheet, identifying the researcher, and explaining the nature and objectives of the research, and a consent form that was signed by the patients, and enabled them to suggest the time of day and telephone number at which they would be prepared to be interviewed.

At all four A\&E departments, recruitment to the study was erratic, owing to a number of factors. Many nurses perform triage; however, analysis of recruitment at one hospital showed that $<30 \%$ of nurses actually recruited a patient, and of those who did, one nurse recruited more than $30 \%$ of the total sample. At another hospital, recruitment was not a gradual process, but a concerted effort to recruit all eligible patients on just three non-consecutive days. Recruitment of patients was less probable during busy periods, such as the holiday season, and during sudden patient influxes when the process of obtaining consent was felt to be prejudicial to the efficient functioning of the department. Higher levels of recruitment were achieved once a cash reward was offered to participating departments. Certain categories of patients, such as girls seeking emergency contraception and persons disagreeably under the influence of drugs or alcohol, were consistently not recruited as a matter of nursing judgement. While the variation in sampling method was not ideal, it is difficult to envisage many other systematic sources of sampling bias. In the hospital in which patients were recruited intensively on 3 days, there was actually a lower proportion of dissatisfied patients than at the other departments.

A total of 352 consents were obtained between 2 July and 2 November 2002. Of these, 321 (91.2\%) were successfully interviewed. More than half of all interviews were conducted in the evenings after the patient had come home from work, and by showing great willingness to defer to the patients' priorities, and to phone back at a time convenient to them, a high level of trust was established. Of the 31 patients not contacted, 18 never answered their telephones (on up to 10 occasions), and/or had changed their telephone provider/ number before interview was attempted, and a further six had consented to their inclusion in spite of having no telephone. Three patients had moved away permanently, three withdrew consent, and one elderly patient was unable to recollect the visit and was excluded. Of the interviews completed, 273 were with the actual patient, 40 with the parent or grandparent of a child, and 8 with the spouse or carer of an adult. In all the non-patient cases, the person interviewed had actually been present in the hospital with the patient.

An electronic database was assembled and analysis performed using SPSS (version 10.1; SPSS, Chicago, IL, USA).

\section{RESULTS}

The sample comprised 176 males and 145 females. There was no significant heterogeneity in sex ratio in the samples from the four hospitals. The age distribution ranged from infancy to 90 years (mean (SD) 36.6 (20.0) years). This is close to a normal distribution, except for a marked excess of patients in the age range 18-22 years. Analysis in the separate hospitals showed this anomaly to be due to a substantial excess of young patients in the university town (hospital D). Mean ages at hospitals D (32.8 years) and C (32.4 years) were significantly lower than at A ( 40.7 years) and B ( 38.33 years) $(\mathrm{p}<0.012)$, and the proportion of patients allocated to triage 5 was higher at hospital D (45\%) than at A (17.1\%), B $(14.3 \%)$, or C $(6.5 \%)(p<00001)$. The excess of triage 5 patients at hospital $\mathrm{D}$ is not a reflection of the student subpopulation that was a characteristic of this sample, but instead appears to reflect a real difference either in the patient mix or in the nurses' application of the Manchester triage categories.

The experiences of patients are quantified for the whole sample. Sex differences and inter-hospital heterogeneity in the patient experience were investigated, but are recorded only where they approached significance.

\section{Why these patients went to A\&E}

Most patients attended A\&E on account of recently occurring illnesses/injury; 251 (78\%) went because of a condition with a recent and sudden onset, $53(16.5 \%)$ for a condition that had occurred recently but become worse, and only 17 (5.5\%) with a problem of a prolonged or chronic nature. For 261 $(82 \%)$ the condition had begun within the preceding week. Injury or suspected injury constituted the predominant reason for attending. Strains, sprains, ligament and tendon damage represent $113(35 \%)$ of the patients, a further 32 $(10 \%)$ proved to have actual fractures, $10(3 \%)$ attended because they suspected they might have cracked a rib, and 6 (2\%) for actual or suspected concussion. Wounds, abrasions, contusions, and crushed digits accounted for $64(20 \%)$, eye 


\begin{tabular}{|c|c|c|c|c|c|c|c|}
\hline \multirow[b]{2}{*}{ Reason } & \multicolumn{3}{|c|}{ West Wales } & \multicolumn{3}{|c|}{ Lancaster } & \multirow{2}{*}{$\begin{array}{l}\text { Comparison } \\
\text { of "reason" } \\
\text { Wales:Lancs } \\
(\%)^{*}\end{array}$} \\
\hline & $n$ & $\%$ & $\begin{array}{l}\text { Median } \\
\mathrm{T}_{\mathrm{u}} \text { (mins) }\end{array}$ & $n$ & $\%$ & $\begin{array}{l}\text { Median } \\
\mathrm{T}_{\mathrm{u}} \text { (mins) }\end{array}$ & \\
\hline $\begin{array}{l}\text { A\&E more appropriate than } \\
\text { GP }\end{array}$ & 83 & 25.9 & 60 & 40 & 17.2 & 817 & 32.5: 32.2 \\
\hline GP would send me anyway & 21 & 6.6 & 90 & 35 & 15.0 & $156 」$ & \\
\hline Referred by GP & 43 & 13.4 & 77.5 & 29 & 12.3 & 360 & 18.1: 23.8 \\
\hline Advised by others than GP & 15 & 4.7 & 30 & 27 & 11.5 & 126 & \\
\hline $\begin{array}{l}\text { Quicker, wait too long for } \\
\text { GP appointment }\end{array}$ & 40 & 12.5 & 90 & 35 & 15.0 & 1387 & \\
\hline More convenient than GP & 26 & 8.1 & 60 & 23 & 10.0 & 48 & 44.4: 42.4 \\
\hline $\begin{array}{l}\text { GP surgery closed/not } \\
\text { available }\end{array}$ & 47 & 14.7 & 60 & 21 & 9.8 & 60 & \\
\hline $\begin{array}{l}\text { No GP/GP more than } 25 \\
\text { miles away }\end{array}$ & 29 & 9.1 & 45 & 18 & 7.6 & $81^{-1}$ & \\
\hline $\begin{array}{l}\text { Already tried GP without } \\
\text { good outcome }\end{array}$ & 15 & 4.7 & 105 & & & & \\
\hline Other & 1 & 0.3 & & 4 & 1.7 & & \\
\hline Total & 320 & 100 & & 233 & 100 & & \\
\hline
\end{tabular}

injuries for $10(3 \%)$, local infections for $17(5 \%)$, foreign bodies (usually splinters) for 9 (3\%), insect stings for 3, and burns for 2. Among the remaining 55 (17\%) were nine systemic infections, six cardiac system conditions, five skin complaints, four genitourinary system conditions, and just two or three patients in each of six other categories. In one region that had inadequate dental cover, dental emergencies self refer in desperation to A\&E, and three such patients were recruited to the sample.

There were 128 patients $(42 \%)$ who became ill or were injured at home, 87 (29\%) injured themselves in the course of recreational activities, $66(22 \%)$ at work, school, or college, and $9(3 \%)$ as a result of road traffic accidents. Among those patients not actually at home at the commencement of their illness, 107 were within 10 miles of home, 48 within 11-100 miles from home, and 17 (of whom 15 were holidaymakers) outside this range.

\section{How the patients travelled to A\&E}

Overwhelmingly, the patients made their way to hospital by private transport: $82(26 \%)$ drove themselves to hospital, 191 $(60 \%)$ travelled as passengers in a private car, and $33(10 \%)$ walked. Public transport and taxi conveyed just 11 people

Table 2 Reason for attending A\&E at this particular time, and utilisation delay times $\left(T_{u}\right)$; this study, and Walsh 1995

\begin{tabular}{|c|c|c|c|c|c|c|}
\hline \multirow[b]{2}{*}{ Reason } & \multicolumn{3}{|c|}{ West Wales } & \multicolumn{3}{|c|}{ Lancaster } \\
\hline & $\mathbf{n}$ & $\%$ & $\begin{array}{l}\text { Median } \\
\mathrm{T}_{\mathrm{u}} \text { (mins) }\end{array}$ & $\mathbf{n}$ & $\%$ & $\begin{array}{l}\text { Median } \\
\mathrm{T}_{\mathrm{u}} \text { (mins) }\end{array}$ \\
\hline $\begin{array}{l}\text { Concern condition } \\
\text { needed seeing now }\end{array}$ & 98 & 30.5 & 30 & 31 & 14.2 & 48 \\
\hline $\begin{array}{l}\text { Left to see if it got } \\
\text { better, it did not }\end{array}$ & 69 & 21.5 & 90 & 21 & 9.6 & 90 \\
\hline $\begin{array}{l}\text { Told to come by } \\
\text { another }\end{array}$ & 67 & 20.9 & 60 & 46 & 21.0 & 75 \\
\hline Pain and discomfort & 46 & 14.3 & 60 & 33 & 15.1 & 72 \\
\hline Convenience & 24 & 7.5 & 150 & 54 & 24.7 & 168 \\
\hline $\begin{array}{l}\text { Thought waiting } \\
\text { times would be less } \\
\text { now }\end{array}$ & 8 & 2.5 & 998 & 17 & 7.7 & 210 \\
\hline Other & 9 & 2.8 & 157 & 17 & 7.7 & 39 \\
\hline Total & 321 & 100 & & 219 & 100 & \\
\hline
\end{tabular}

(3.5\%), and three individuals travelled by ambulance or ambulance car. In the latter cases, the ambulance had been summoned by, or at the suggestion of, their GP. One significant sex difference emerged; men are twice as likely as women to drive themselves to hospital when in need of medical attention. Some of these acknowledged the hazardous nature of this decision; however, in a rural area, there may be no viable alternative, and in this population the decision to summon an ambulance is not taken lightly. Indeed, the discursive interviews often indicated the patients' reliance on friends or relatives for transport, and the delays endured to avoid making demands upon an on call doctor or the ambulance service. These voluntary networks are adequate to the needs of most patients: 252 (78\%) said that there were "no real obstacles" to getting themselves to hospital, $37(11.5 \%)$ had considered transport to be a problem, $20(6.2 \%)$ delayed attendance because of other work related responsibilities, and $9(2.8 \%)$ because of their responsibility for other dependants. Women were more likely to cite transport, and men to cite work responsibilities as obstacles to their attendance. Once a means of transport had been selected, times and distances were rarely excessive. The average patient travelled 7.4 miles and took 16 minutes to travel to hospital. With the exception of one local who worked away, and travelled 100 miles home to go to the A\&E department there, the range of journey time and distance did not exceed 40 miles or 1 hour.

Actual travel time is, however, only a component part of the time encompassed by decision making and action. Following the analysis by Walsh ${ }^{21}$ the utilisation delay time $\mathrm{T}_{\mathrm{u}}$ (time elapsed from decision to registration) was computed. This variable is strongly skewed (138 patients made their way to hospital in less than an hour), the median is 1 hour, and the maximum postponement was 24 hours. Notwithstanding the more rural situation, median utilisation delay in this sample was shorter, in most situations, than in Walsh's urban sample (tables 1 and 2).

\section{Deciding to attend}

A number of linked questions were designed to elucidate, without being critical or judgemental, how the patient decided to go to A\&E, what sources of advice they may have drawn upon in reaching their decision, and whether they considered using or involving their GP in the process. As was to be expected with the largely indigenous nature of the 
Table 3 Dissatisfaction levels in relation to whether the patient received treatment

\begin{tabular}{|c|c|c|c|c|}
\hline & $\begin{array}{l}\text { Mean } \\
\text { score* }^{*}\end{array}$ & n & SE & $95 \% \mathrm{Cl}$ \\
\hline No treatment & 9.18 & 50 & 0.591 & 10.33 to 8.02 \\
\hline Any treatment & 7.76 & 269 & 0.219 & 8.19 to 7.33 \\
\hline $\begin{array}{l}\text { Analysis of } \\
\text { variance }\end{array}$ & $F=6.264$ & d.f. 1 & $p<0.013$ & \\
\hline
\end{tabular}

*For dissatisfaction. SE, standard error; Cl, confidence interval.

sample, 289 (90\%) of the patients were registered with a local GP. Of the remainder, 29 (9\%) were registered with a GP but not locally, and three had no GP arrangements, being army personnel or foreign visitors. Of the sample, 191 (59.5\%) went directly to the A\&E department, and almost half of these considered that their injury was so obviously appropriate to A\&E care that they sought no advice in reaching that decision. A further $71(22 \%)$ first sought the advice of their GP or health centre, either by telephone or in person, and then made their way to A\&E, while 59 (18.5\%) replied that they had considered but rejected calling upon primary care.

In an open ended inquiry as to why they decided to use A\&E, it emerged that patients' replies reflected the view that the GP service could not meet their needs (table 1): 104 (32.5\%) felt that A\&E was the right place to go, or that the GP would send them there anyway; 58 (18\%) were actually referred by their GP, or were advised to go by other health workers or first aiders. For many others, the GP service was not perceived as a viable option on account of inaccessibility through time or distance. Together, these categories account for 141 patients (44\% of the sample). While it is often commented in the profession that people use A\&E as a way of gaining access to a "second opinion", having tried their GP without a good outcome, this was true of just 15 patients (4.7\% of the sample). These findings are remarkably similar to the findings of Walsh ${ }^{21}$ in an interview study of ambulatory minor injuries patients in Lancaster (table 1).

While almost half the sample had taken no advice in reaching their decision to attend hospital, 172 stated they had been advised by various people: family and friends (62), work/school colleagues (21), GPs (37), receptionists or first aiders (43), police (2), or pharmacist (1). Patients were specifically asked whether they had used NHS Direct: just six had done so, all of whom were women; two were mothers acting for young infants, and three other patients aged 1720 years. NHS Direct was launched in Wales in April 2002.

Most frequently, the timing of the visit to A\&E was on account of the patient's perception that the condition demanded immediate attention or that they had waited in the hope that it would get better, and it had not. A comparison with the same question asked by Walsh ${ }^{21}$ (table 2) shows that this sense of urgency was more commonly the reason in the Welsh sample, while patients' convenience more frequently dictated the time of attendance among the Lancaster patients. In both studies, a sense of urgency is accompanied by shorter utilisation delays $\left(\mathrm{T}_{\mathrm{u}}\right)$.

\section{Feelings in anticipation}

Patients were invited to estimate, on a scale ranging from (1) "very" to (5) "not at all" their levels of anxiety, the urgency they felt their condition merited, and the levels of pain they were suffering before they were attended to: $100(31 \%)$ scored their anxiety and 97 (30\%) scored the urgency of their case in the two highest categories (these variables are strongly correlated; Pearson's product moment correlation $0.518, \mathrm{p}<0.001)$, while $85(26 \%)$ described themselves as not at all anxious, and $77(24 \%)$ as not urgent. Pain was more frequently an issue, with 140 (44\%) putting their pain levels in the top two categories, and just 46 (14\%) being able to wait without experiencing pain. These variables were combined to form a composite score representing the stress level of the patient awaiting treatment, with a range of 3-15 (mean 9.36, SD 2.24) and a normal distribution. Neither age nor sex showed any correlation with reported stress.

\section{Experience of treatment}

Patients were encouraged to describe their experience of the hospital visit and then to allocate scores ranging from 1 (very satisfied) to 5 (not at all satisfied) to their views of the service on five separate criteria. These were satisfaction with doctors, nurses, facilities, speed, and treatment. These scores were also added together, to provide a composite score representing overall satisfaction (range 5-25).

As would be expected, the patients in this sample showed a diurnal attendance pattern, only four arriving between 2200 and 0700. After treatment, 311 went home, seven were admitted for <24 hours, and three were admitted or transferred with resultant stays of 4-9 days. Time spent in A\&E from registration to departure, according to patients' own estimates, was <1 hour: 144 (46\%), 1-2 hours: 105 (34\%), 2-3 hours: 34 (11\%), 3-4 hoursL: 22 (7\%), and 45 hours $6(2 \%)$. One patient with a fracture waited 7 hours on a morphine infusion before having it set and being admitted to a ward.

It is consistent with the types of illnesses that the commonest intervention was an $x$ ray (128 patients; $40.1 \%)$. A plaster cast was used for $23(7.2 \%)$, while a further $70(22.3 \%)$ went home with a strapping, sling, or support; 20 $(6.2 \%)$ received stitches or wound closures and $41(12.8 \%)$ wound dressings; 96 (30\%) were provided with some tablet medication to go home with, most commonly anti-inflammatories or antibiotics; $52(16 \%)$ were instructed to return to the A\&E clinic and $55(17 \%)$ to seek follow up attention from their GP; and $50(15.6 \%)$ received no treatment other than assessment and advice.

The modal response of patients on all five measures of satisfaction was "very satisfied". This response was given in respect of doctors $(206 ; 66 \%)$, nurses $(239 ; 76 \%)$, facilities $(207 ; 65 \%)$, speed of treatment $(199 ; 63 \%)$ and quality of treatment $(220 ; 71 \%)$. The highest score on all five criteria was awarded by $118(38 \%)$ of the patients, and a sharply declining proportion expressed increasing levels of dissatisfaction. An investigation into the factors predictive of dissatisfaction showed some trends. There was a weak correlation between the stress levels reported by patients and the overall level of satisfaction (Kendall's $\tau$ 0.092, Spearman's correlation coefficient 0.122, $\mathrm{p}<0$ 05). Those under higher stress of pain or anxiety were less satisfied with care. Satisfaction levels were similar in subsamples that received any category of intervention and treatment; only those 50 patients who received no $x$ ray, plasters, strappings, dressings, biochemical tests, or medication were significantly less satisfied than other groups (table 3). However, although these patients had a lower mean level of satisfaction than did those who received some form of treatment, going to hospital and leaving with nothing other than a doctor's opinion and advice still elicited maximum satisfaction in 19/50 (30\%) of patients.

At the end of the interview patients were asked whether, in similar circumstances in the future, they would take the same or a different course of action in obtaining healthcare; $209(66 \%)$ would do the same again, $79(25 \%)$ would go to their GP, 20 (6\%) would not bother, and 5 (1.5\%) would go to a different hospital. These responses correlated with rising 
median levels of dissatisfaction with the service that they received on their visit.

\section{Dissatisfied patients}

While the modal response of patients on all five criteria is one of satisfaction, there were also less satisfied patients and a small number of very unhappy customers. However, only one patient was planning to make an official complaint. Most patients were happy to express their views, whether positive or negative, but would not have done so unless asked.

A qualitative analysis of the 87 patients whose composite satisfaction score was 10 or higher revealed important trends and also different patterns within the four participating A\&E departments.

Overall, the most significant feature in determining patient dissatisfaction was the social skills of the doctor: 48 patients ( $55 \%$ of this 87 ) cited doctor behaviour in their comments. In these encounters doctors were often described as "offhand", "dismissive", "rude", "arrogant", "abrupt", or "unsympathetic". Some patients felt they were treated as time wasters, or that the doctor did not take the time to explain the diagnosis. Sometimes a doctor did not return to discuss findings of, for example, an $x$ ray, but simply sent a nurse in to apply a bandage. A smaller number of comments about doctors related to patients' unease at the poor language skills of foreign doctors. Despite these criticisms, only 13 of these 48 patients considered themselves "not at all satisfied" with the actual treatment they had received.

Dissatisfaction with treatment tended to be an issue where junior doctors appeared inexperienced or unable to perform a procedure successfully. Splinters seemed to be a particular problem, and patients felt there was reluctance to perform minor surgery: one patient was transferred to another hospital, another sent home with antibiotics and then sent back, with a letter from his GP, before surgery was performed. Patients with muscular spasm or back pain often felt dissatisfied in retrospect at how little help their visit to A\&E had been, and related how readily the problem was subsequently sorted out by manipulation by an osteopath or chiropractor.

Facilities were criticised most frequently in the least modern of the departments, for reasons such as awkward swing doors, cold waiting areas, insufficient seating, inadequate facilities for babies and young children, or items of equipment that were not working. Parking within an acceptable distance from A\&E was a problem, especially for the elderly, or for parents carrying children. Direction signs to A\&E, although no problem for locals, were found to be woefully inadequate by some strangers to the area.

In general, nurses were well liked, and in three of the four hospitals attracted very few adverse comments. Patients gain much support from friendly and supportive nurses, and indeed, this often ameliorates their displeasure at other shortcomings. At the fourth hospital, patients were more critical of their nursing care. Here, $13 / 31$ (42\% of the dissatisfied patients) felt that they had been placed in curtained cubicles and left alone, sometimes for long periods of time (mean waiting time 2.7 hours, range 2-4 hours, $\mathrm{n}=13$ ). In the study as a whole, patient dissatisfaction was not significantly correlated with actual length of wait, (Pearson product moment correlation 0.068, $\mathrm{n}=320$, NS) but it rose to significance in this hospital among patients who felt less than fully satisfied with attention they had received from nurses (Pearson product moment correlation 0.42, $\mathrm{n}=24, \mathrm{p}<0.05)$. Patients who felt they had received this kind of discrimination included young adults whose injuries had been associated with partying or fighting (even if they were the victim), holidaymakers, and people with acute dental pain.

\section{DISCUSSION}

This study paints a picture of the characteristics of the less urgent patients at four Welsh A\&E departments. Approximate figures for the annual attendances (2002-03) at each of the A\&E departments, with percentages triaged 4 and 5 as a combined figure in brackets, were: 38000 (66\%), $28000(54 \%), 29000(55 \%)$, and 19000 (57\%). Three of these hospitals serve very rural areas, and one serves a predominantly urban and deprived area and its rural hinterland.

Waiting times are, in comparison with many city hospitals, extremely good, and patients take responsibility for their own transport needs. There is no evidence of inappropriate calling out of the emergency services.

Comparisons with a similar urban English study show that the reasons for which the patients elected to attend A\&E were broadly similar in both settings. Despite the dispersed population, Welsh patients generally got themselves to hospital in less time than their urban counterparts, and fewer Welsh patients selected their time of attendance for reasons of personal convenience. Moreover, very few Welsh patients can usefully be considered "inappropriate attenders" once their circumstances and anxieties are taken into account. The few whose symptoms least justified A\&E attendance were predominantly health service workers, who popped into A\&E as an alternative to taking time off to go to their GP. It is probably to the advantage of the hospitals in which they work that they did so.

Patients from this overwhelmingly local population do not have unreasonably high expectations, are reluctant to make demands, and are grateful and pleased with the attention that they receive. The cost-benefit assessments, which were shown by Walsh ${ }^{21}$ to underlie patient decision making about seeking health care at A\&E, also apply to this study. While some patients could, theoretically, have been treated by their GP, the perceived difficulties with GP services and problems in obtaining out of hours attention, combined with a tendency in some practices for patients to be redirected to A\&E by the receptionist, makes change unlikely. Even an improvement of GP accessibility might have a less than dramatic effect upon patients' decisions. An analysis at Sheffield of the reasons underlying low priority patients' decisions to attend A\&E concluded that increasing availability of other services (general practice, minor injuries units, walk in centres, and NHS Direct) is unlikely to have much impact on the demand for A\&E services. ${ }^{25}$

The small number of patients who used NHS Direct reflects the very recent introduction of the service in Wales. As was also shown in a West Yorkshire study, ${ }^{26}$ patients who did use NHS Direct were younger, and although all appreciated the advice, they would probably have taken the same course of action were it not available.

The culture of the Welsh rural population is one of extreme reluctance to criticise authority, and indeed many people believe that complaint will be detrimental to their own benefit. While there is no doubt that the picture of A\&E provision is predominantly positive, and a matter for satisfaction, it should be noted that the official complaints procedure is unlikely to be an adequate tool for judging the experience of patients. Even among patients with serious issues, most feel relieved that their adverse experience is at an end, and are unlikely to make their opinions known unless asked.

The culture of A\&E tends to magnify the significance of incidents of unreasonable patient behaviour or expectations in the retelling, such that these become magnified to the status of "urban legends". This culture in turn may lead to the unnecessarily negative experiences reported by a minority of patients. As the NHS Modernisation Agency ${ }^{27}$ now 
champions replacing the triage process with a "see and treat" approach to patient care in A\&E, the continued survival of the stereotype of the "inappropriate attender" can only be an obstacle to the implementation of the new way of working.

\section{ACKNOWLEDGEMENTS}

This work was commissioned and funded by the Dyfed Research and Development Consortium (comprising the Carmarthenshire, Ceredigion, and Mid-Wales, and the Pembrokeshire and Derwen NHS Trusts) through the provider support funding stream from the Welsh Assembly Government. Thanks are due to $\mathrm{K}$ Bashir, J Williams, M Vaziri, M Jones, A Davies, C Edwards, S Hughes, R Izzard, L Jenkins, and many other staff members at the A\&E departments, and to C Hayes, L Seale, and B Smith who were part of the steering committee overseeing this project. L Seale and B Smith also proofread the manuscript in detail.

\section{Authors' affiliations}

C D Palmer, K H Jones, Dyfed R\&D Consortium, Wales

P A Jones, S V Polacarz, G W L Evans, Pembrokeshire \& Derwen NHS Trust, Wales

Competing interests: none declared

\section{REFERENCES}

1 Dale J. Primary care: the old bugbear of accident and emergency services. BrJ Gen Pract 1992;42:90-1.

2 Tomlinson B. Report on the enquiry into London's health service, medical education and research. London: HMSO, 1992.

3 Dale J, Green J, Reid F, et al. Primary care in the Accident and Emergency department. I. Prospective identification of patients. BMJ 1995;311:423-6.

4 Dale J, Green J, Reid F, et al. Primary care in the Accident and Emergency department. II. Comparison of general practitioners and hospital doctors. BMJ 1995;311:427-30.

5 Dale J, Lang H, Roberts JA, et al. Cost effectiveness of treating primary care patients in accident and emergency: a comparison between general practitioners, senior house officers and registrars. BMJ 1996;312:1340-4.

6 Freeman G, Meakin R, Lawrenson R, et al. Primary care units in A\&E departments in North Thames in the 1990s: initial experience and future implication. Br J Gen Pract 1999;49:107-10.

7 Stoddart D, Ireland AJ, Crawford R, et al. Impact on an accident and emergency department of Glasgow's new primary care emergency service. Health Bull 1999;57:186-91.

8 Murphy AW, Bury G, Plunkett PK, et al. Randomised controlled trial of general practitioner versus usual medical care in an urban accident and emergency department: process, outcome and comparative cost. $B M$ 1996;312:1 135-42.

9 Avery AJ, Groom L, Boot D, et al. What problems do patients present with outside normal general practice surgery hours? A prospective study of the use of general practice and accident and emergency services. J Public Health Med 1999;21:88-94.

10 Murphy AW. "Inappropriate" attenders at accident and emergency departments II: health service responses. Fam Pract 1998;15:33-7.

11 Hull SA, Jones IR, Moser K. Factors influencing the attendance rate at accident and emergency departments in East London: the contributions of practice organization, population characteristics and distance. J Health Serv Res Policy 1997;2:6-13

12 Jankowski RF, Mandalia S. Comparison of attendance and emergency admission patterns at accident and emergency departments in and out of London. BMJ 1993;306:1241-3.

13 Carlisle R, Groom LM, Avery AJ, et al. Relation of out of hours activity by general practice and accident and emergency services with deprivation in Nottingham: longitudinal survey. BMJ 1998;316:520-3.

14 Dale J, Dolan B. Do patients use minor injury units appropriately? J Public Health Med 1996;18:152-6.

15 Campbell JL, General practitioner appointment systems, patient satisfaction, and use of accident and emergency services: a study in one geographical area. Fam Pract 1994; 11:438-45.

16 Wise M. Inappropriate attendance in Accident and Emergency. Accid Emerg Nurs 1997;5:102-6.

17 Shelton E. Hospital doctor 2002. Accessed 14 March 2003. Available at: http://www.doctor.net.

18 Wolcot B. What is an emergency? Depends on whom you ask. J Am Coll Emerg Physician 1979;8:233-41.

19 Platt H. National Health Services accident and emergency services. London: HMSO (HM68, 83), 1962

20 Dyfed-Powys A\&E Network Nursing Audit. Findings presented at Network meeting. Bronglais General Hospital, 12 September 2002.

21 Walsh $M$. The health belief model and use of accident and emergency services by the general public. J Adv Nur 1995;22:694-9.

22 Murphy AW. 'Inappropriate' attenders at accident and emergency departments I: definition, incidence and reasons for attendance. Fam Pract 1998;15:23-32.

23 Saunders J. A review of health professional attitudes and patient perceptions on "inappropriate" accident and emergency attendances. The implications for current minor injury service provision in England and Wales. J Adv Nurs 2000;31:1097-105.

24 Audit Commission. By accident or design: improving A\&E Services in England and Wales. London: HMSO, 1996.

25 Coleman P, Irons R, Nicholl J. Will alternative immediate care services reduce demand for non-urgent treatment at accident and emergency? Emerg Med J 2001;18:482-7.

26 Gaffney P, Crane S, Johnson G, et al. An analysis of calls referred to the emergency 999 service by NHS Direct. Emerg Med J 2001;18:302-4.

27 Castille K. See and treat. London: NHS Modernisation Agency, 2002. Available at: http://www.modern.nhs.uk/. 